\title{
Classification of Pneumoconiosis on HRCT Images for Computer-Aided Diagnosis
}

\author{
${\text { Wei } \text { ZHAO }^{\dagger a)} \text {, Rui XU }}^{\dagger}$, Yasushi HIRANO ${ }^{\dagger}$, Rie TACHIBANA ${ }^{\dagger \dagger}$, Shoji KIDO ${ }^{\dagger}$, Members, \\ and Narufumi SUGANUMA ${ }^{\dagger \dagger \dagger}$, Nonmember
}

\begin{abstract}
SUMMARY This paper describes a computer-aided diagnosis (CAD) method to classify pneumoconiosis on HRCT images. In Japan, the pneumoconiosis is divided into 4 types according to the density of nodules: Type 1 (no nodules), Type 2 (few small nodules), Type 3-a (numerous small nodules) and Type 3-b (numerous small nodules and presence of large nodules). Because most pneumoconiotic nodules are small-sized and irregular-shape, only few nodules can be detected by conventional nodule extraction methods, which would affect the classification of pneumoconiosis. To improve the performance of nodule extraction, we proposed a filter based on analysis the eigenvalues of Hessian matrix. The classification of pneumoconiosis is performed in the following steps: Firstly the large-sized nodules were extracted and cases of type 3-b were recognized. Secondly, for the rest cases, the small nodules were detected and false positives were eliminated. Thirdly we adopted a bag-of-features-based method to generate input vectors for a support vector machine (SVM) classifier. Finally cases of type 1,2 and 3-a were classified. The proposed method was evaluated on 175 HRCT scans of 112 subjects. The average accuracy of classification is $90.6 \%$. Experimental result shows that our method would be helpful to classify pneumoconiosis on HRCT.

key words: pneumoconiosis, computer-aided diagnosis, HRCT, Hessian matrix, bag-of-features
\end{abstract}

\section{Introduction}

Pneumoconiosis refers to a group of occupational diseases caused by inhalation and retention of dust particles in the lung. After years of inhaling dust, the pneumoconiosis would lead to a severe lung function impairment, especially in the smokers. At present there are a large number of pneumoconiosis patients in the developing countries. For example, in China, it is reported that the pneumoconiosis is the most serious occupational diseases and there are 23,812 new cases in 2010 [1].

According to the criterion made by International Labour Organization (ILO), the pneumoconiosis is diagnosed based on the profusion (severity) of nodular opacities on the chest X-ray (CXR) radiographs. However, it is suggested that the high-resolution computed tomography

Manuscript received June 15, 2012.

Manuscript revised September 18, 2012.

${ }^{\dagger}$ The authors are with the Applied Medical Engineering Science, Graduate School of Medicine, Yamaguchi University, Ubeshi, 755-8611 Japan.

The author is with Information Science and Technology Dept., Oshima National College of Maritime Technology, Yamaguchi-ken, 742-2193 Japan.

${ }^{*}+{ }^{+}$The author is with the Department of Environmental Medicine, Kochi Medical School, Kochi University, Nankoku-shi, 783-8505 Japan.

a)E-mail: r501ug @yamaguchi-u.ac.jp

DOI: 10.1587 /transinf.E96.D.836
(HRCT) is better for diagnosis of pneumoconiosis because the HRCT can detect nodules which would be overlapped with ribs and vessels on the CXR radiographs [2]. Because the diagnosis of pneumoconiosis mainly depends on individual radiologists' experiences. The subjective differences may lead misdiagnosis for patients. Additionally, the vast amount of axial images in each HRCT scan place a great burden on radiologists. Therefore, computer-aided diagnosis (CAD) method is required to give a second-opinion and reduce burden for radiologists. Although several CAD schemes utilizing CXRs have been developed over the last two decades [3]-[7]. According to our knowledge, there was no research on CAD of pneumoconiosis on HRCT images.

In this research, we proposed a CAD method to classify pneumoconiosis on HRCT images according to Japanese Pneumoconiosis Law. In Japan, the pneumoconiosis is divided into 4 types based on the density and diameter of nodular opacities: Type 1 (no nodules), Type 2 (few small nodules (diameter $\leq 10 \mathrm{~mm}$ )), Type 3 -a (numerous small nodules) and Type 3-b (numerous small nodules and presence of larger nodules (diameter $>10 \mathrm{~mm}$ )). Figure 1 illustrates HRCT images of 4 types of pneumoconiosis. Although the main image findings of lung cancer and pneumoconiosis are both pulmonary nodules, the lung cancer of-

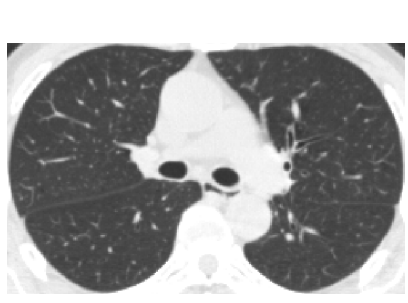

(a)

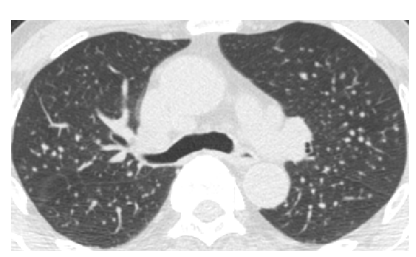

(c)

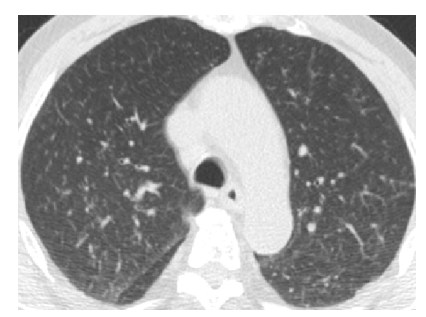

(b)

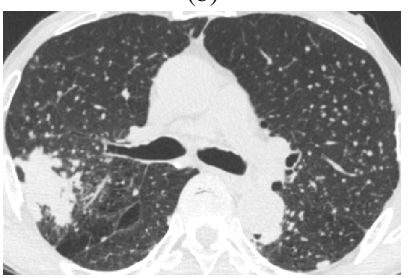

(d)
Fig. 1 HRCT images of pneumoconiosis and lung cancer: (a) Type 1, absence of nodule; (b) Type 2, few small nodules; (c) Type 3-a, numerous small nodules; (d) Type 3-b, numerous small nodules and presence of larger nodules. 
ten appears one single or several solitary pulmonary nodular lesions (SPNs). The pneumoconiosis is typical of diffuse nodular opacities of various sizes and shapes spreading wide regions inside lungs. The required conditions of CAD systems for these are different either. A lung cancer CAD system is focused on locating SPNs and differentiating malignant SPNs from benign ones [9]-[12]. However, the proposed CAD systems of pneumoconiosis is aimed to classify the four types of pneumoconiosis in order to facilitate the diagnosis for radiologists. Considering that the pneumoconiosis of type 3-b and non-3-b (type 1, 2 and 3-a) is diagnosed based on the size of nodules, we recognized the cases in two-steps: 1.Recognizing cases of type 3-b from all scans, 2.Classifying cases of type 1,2 and 3-a from the rest scans. Because numerous pneumoconiotic nodules are small-sized and irregular-shape, only few nodules could be extracted by conventional methods in the CAD systems of pneumoconiosis. It may affect the classification of pneumoconiosis. Therefore, we should adopt a novel filter to detect nodule candidates as many as possible at first, and then eliminate false positives.

The proposed method was tested on 175 HRCT scans of 112 different subjects. The scans are divided into two completely independent data sets. The training set including 90 scans is used to train the algorithms and the test set including 85 scans is adopted to evaluate performance of proposed method. There are three main contributions in this study: 1.Developing the first CAD scheme to classify pneumoconiosis on HRCT images; 2.Designing a novel nodule filter according to the characteristic of pneumoconiotic nodules; 3.Adopting a bag-of-features-based method in the classification of pneumoconiosis. The paper is organized as follows. In Sect. 2, we describe the framework of proposed CAD method. The experimental results are given and discussed in Sect. 3. Finally we conclude the paper in Sect. 4.

\section{Method}

Figure 2 illustrates the framework of the proposed CAD method. Due to the fact that the cases with large-sized nodules are diagnosed as type 3-b, we recognized the cases of type 3-b at first, and then classified the rest cases. Firstly the large-sized nodules were detected from all scans. Secondly the cases of type 3-b were recognized based on the size of nodules. Thirdly the small-sized nodules were extracted from the rest cases. In the fourth place we adopted a bag-of-features-based method to generate input vectors for a support vector machine (SVM) classifier. Finally the cases of type 1,2 and 3-a were classified.

\subsection{Nodule Extraction}

The filters based on analysis of eigenvalues of Hessian matrix have been developed to enhance blob-like objects [11], [12]. Table 1 summarizes the conditions of eigenvalues in local structures of various shapes, where $\lambda_{1}, \lambda_{2}$ and $\lambda_{3}$ represent the eigenvalues of Hessian matrix $\left(\lambda_{3} \leq \lambda_{2} \leq \lambda_{1}\right)$.

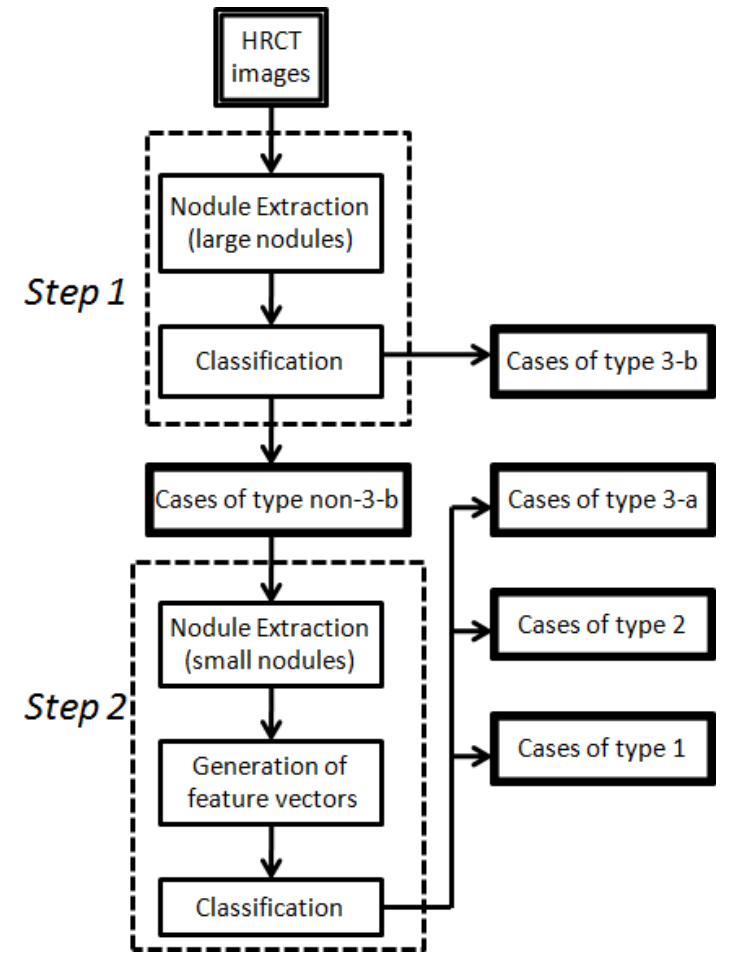

Fig. 2 Overall scheme of proposed CAD method.

Table 1 Eigenvalue condition of local structures, $\lambda_{1}, \lambda_{2}, \lambda_{3}$ represent the eigenvalues of Hessian matrix [11].

\begin{tabular}{|c|c|}
\hline Structures & Eigenvalue condition \\
\hline Nodule & $\lambda_{3} \approx \lambda_{2} \approx \lambda_{1} \ll 0$ \\
\hline Vessel & $\lambda_{3} \approx \lambda_{2} \ll \lambda_{1} \approx 0$ \\
\hline Cortex & $\lambda_{3} \ll \lambda_{2} \approx \lambda_{1} \approx 0$ \\
\hline
\end{tabular}

Because of the various conditions of eigenvalues of Hessian matrix, the Hessian-based filter can enhance the nodules while suppress objects of other shapes. In order to enhance the nodules of various scales, the filters are convolved with Gaussian kernels. By adjusting the standard deviation of Gaussian convolution, the objects with specific range of sizes can be enhanced.

Sato defined a nodule filter as [11]

$$
S_{\text {blob }}= \begin{cases}\left|\lambda_{3}\right|\left(\frac{\lambda_{2}}{\lambda_{3}}\right)^{\gamma}\left(\frac{\lambda_{1}}{\lambda_{2}}\right)^{\gamma}, & \lambda_{3} \leq \lambda_{2} \leq \lambda_{1}<0 \\ 0, & \text { otherwise }\end{cases}
$$

which can be simplified as $-\lambda_{1}$ when $\gamma$ is fixed to 1 , one of preferred value. Figure 3 (a) illustrates the color-code result of nodule enhancement by Sato's filter. The values of standard variation were fixed to $0.6,1,1.5$ and 2 pixels. When the response increases, the color will gradually change from blue by yellow to red. This method has good performance on discriminating nodules from other tissues, but the response of tiny nodules is as weak as false positives. Figure 3 (b) shows the result of nodule extraction after thresholding. The threshold is called nodular threshold in this paper. Numerous nodules were removed. In [12] Li designed his nodule filter as 


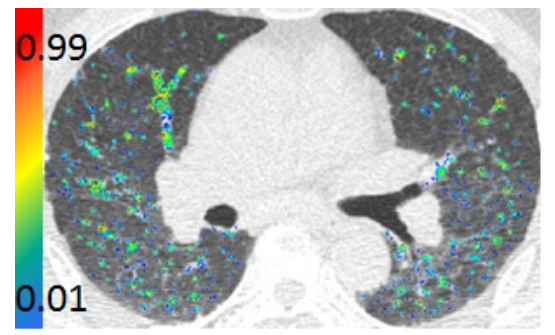

(a)

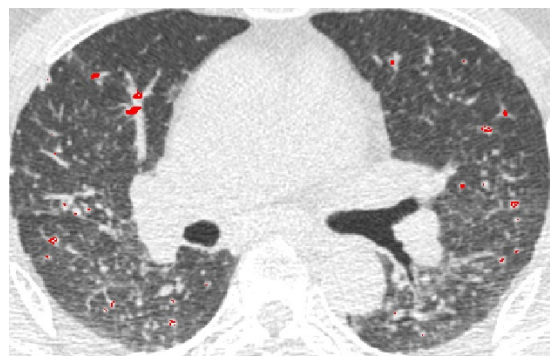

(d)

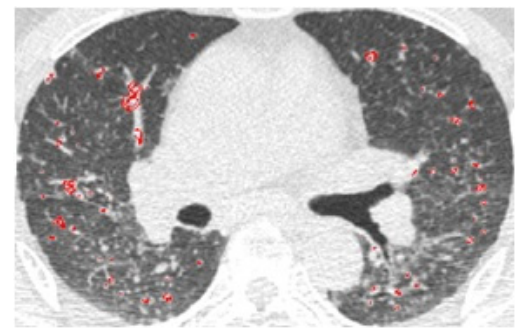

(b)

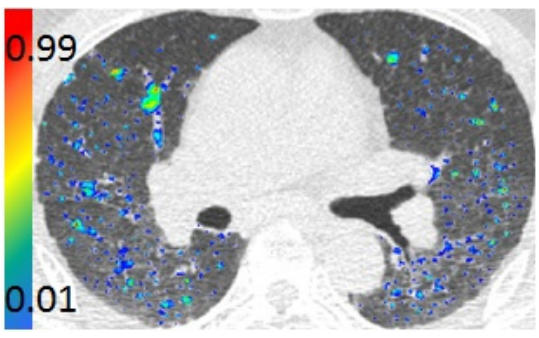

(c)

Response

Fig. 3 Result of nodule extraction by conventional methods: (a) Enhancement of small nodules by Sato's filter; (b) Thresholding of (a) (the nodular threshold $=0.5$ ) (c) Enhancement of small nodules by Li's filter; (d) Thresholding of (c) (the nodular threshold $=0.5$ ); (e) Comparison of response between Sato's and Li's filter.

$$
L_{\text {blob }}= \begin{cases}\left|\lambda_{1}\right|^{2} /\left|\lambda_{3}\right|, & \lambda_{3} \leq \lambda_{2} \leq \lambda_{1}<0 \\ 0, & \text { otherwise }\end{cases}
$$

Figure 3 (c) shows the result of nodule enhancement by this filter, and Fig. 3 (d) gives the result of nodule extraction by Li's filter. It can be found that the number of nodules is further decreased after thresholding. The two conventional Hessian-based filters are compared in Fig. 3 (e). It is clearly that the response of Sato's filter descends slower than Li's filter. So the small-sized nodules can be given a stronger response whose magnitude of eigenvalues is relative small, and greater number of nodules can be extracted after thresholding. It can be deduced that the slower descending response is advantageous to detect more pulmonary nodules.

To improve the performance of extracting pneumoconiotic nodules, we firstly obtained nodule-shaped objects as many as possible, then eliminated false positives, i.e. vascular intersections. Considering that the eigenvalues are approximately equal in the nodules' center, we adopted an monotonically increasing function to represent the difference between the eigenvalues $\lambda_{i}$ and $\lambda_{j}$,

$$
f\left(\lambda_{i}, \lambda_{j}\right)=\frac{2\left(\lambda_{i} / \lambda_{j}\right)}{1+\left(\lambda_{i} / \lambda_{j}\right)^{2}}
$$

When $\lambda_{i}=\lambda_{j}$, the function comes to its maximum. The output of our nodule filter is defined as the product of differences between $\left(\lambda_{1}, \lambda_{2}\right)$ and $\left(\lambda_{2}, \lambda_{3}\right)$

$$
\begin{aligned}
f_{\text {blob }} & =f\left(\lambda_{1}, \lambda_{2}\right) \times f\left(\lambda_{2}, \lambda_{3}\right) \\
& = \begin{cases}\frac{4\left(\lambda_{1} / \lambda_{3}\right)}{1+\left(\frac{\lambda_{1}}{\lambda_{2}}\right)^{2}+\left(\frac{\lambda_{2}}{\lambda_{3}}\right)^{2}+\left(\frac{\lambda_{1}}{\lambda_{3}}\right)^{2}}, & \lambda_{3} \leq \lambda_{2} \leq \lambda_{1}<0 \\
0, & \text { otherwise }\end{cases}
\end{aligned}
$$

Figure 4 (a) gives the response of our nodule filter. The less the difference between eigenvalues, the greater will be response. When the eigenvalues meet the condition $\lambda_{1} \approx \lambda_{2} \approx$ $\lambda_{3}$, in other words, the voxel is located in the nodules' center, the response of proposed nodule filter reaches its maximum. When the differences between eigenvalues are increasing, the response of the filter descends. Figure 4 (b) illustrates the result of nodule enhancement by proposed filter. Figure 4 (c) shows the result of nodule extraction after thresholding. It can be found that the proposed filter can detect more small-sized nodules than the conventional filters. Figure 4 (d) shows the result of enhance large-sized nodules, the selected scales were 4 and 5 pixels. Considering that the scales of most false positives are less than the large-sized nodules, the detected large nodules can be directly adopted in experiment.

It is inevitable that there are numerous false positives in the result of extracting small nodules, for example, the vascular intersections whose condition of eigenvalues is similar with nodules. We designed a vessel filter to remove the false positives meanwhile preserve nodules as many as possible. Since the condition of eigenvalues in vessels' center is $\lambda_{3} \approx \lambda_{2} \ll \lambda_{1} \approx 0$ [11], when local voxel locates in the center line of vessel, the response of proposed vessel filter should come to its maximum. The vessel filter is defined as:

$$
f_{\text {line }}= \begin{cases}\exp \left[-\left(\frac{\lambda_{1}}{\lambda_{2}}\right)^{1 / 2}\right] \times \frac{2 \frac{\lambda_{2}}{\lambda_{3}}}{1+\left(\frac{\lambda_{2}}{\lambda_{3}}\right)^{2}}, & \lambda_{3} \leq \lambda_{2}<0, \lambda_{3} \leq \lambda_{2} \leq \lambda_{1} \\ 0, & \text { otherwise }\end{cases}
$$




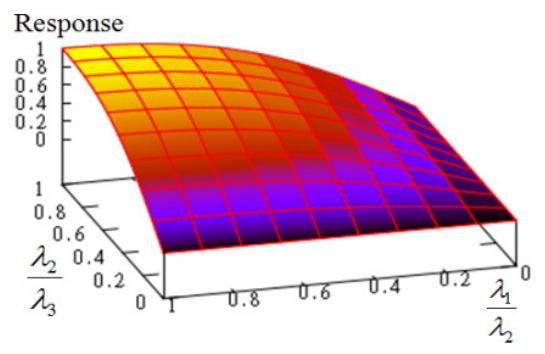

(a)

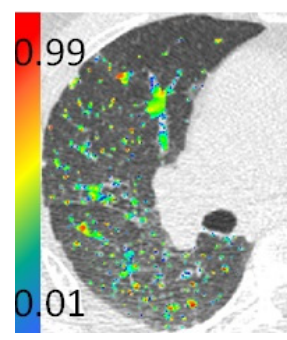

(b)

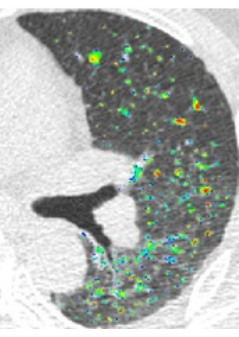

Response

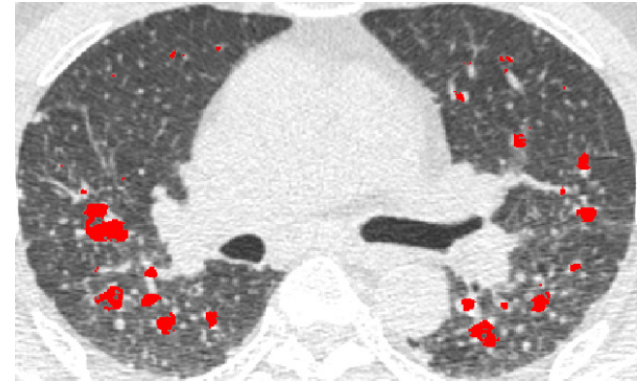

(d)

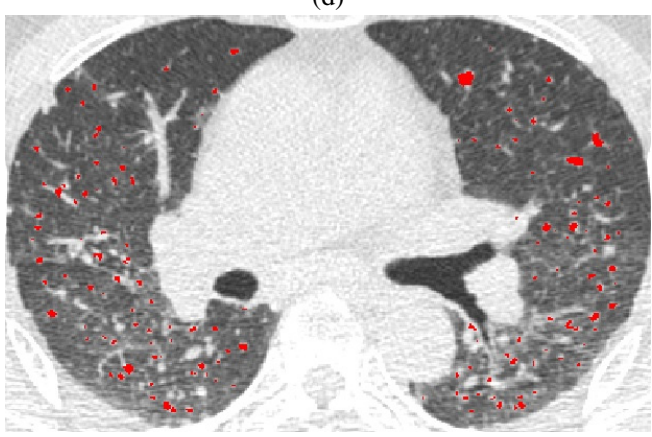

(f)

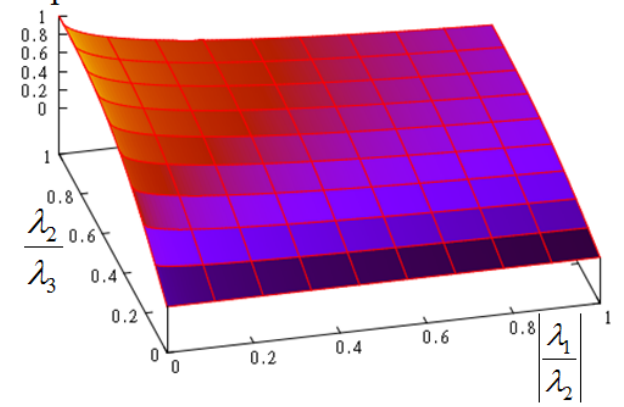

(e)

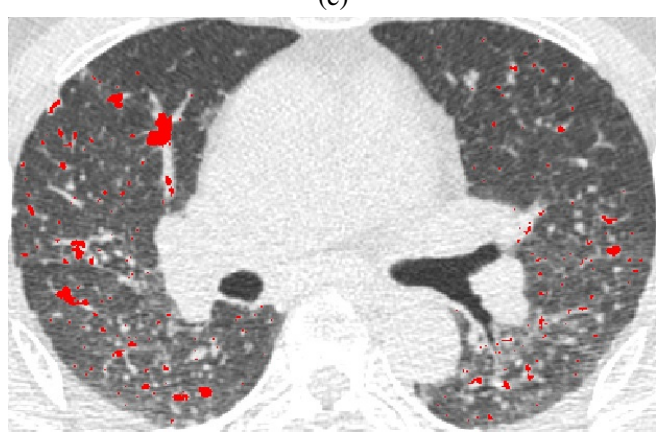

(g)

Fig. 4 Result of nodule extraction: (a) Response of nodule filter; (b) Multiscale enhancement of small nodules with scales of $0.6,1,1.5$, and 2 voxels; (c) Thresholding of (b) (the nodular threshold $=0.5$ ); (d) Result of detecting large nodules, the selected scales are 4 and 5 voxels; (e) Response of our vessel filter; (f) Result of subtracting false positives from (c) (the vascular threshold = 0.7); (g) The removed false positives from (c).

when local voxel locates in the center line of vessel, the response of proposed vessel filter comes to its maximum. The false positives are removed as follows. 1.Thresholding the response of nodule filter and vessel filter respectively. 2.Removing the objects which exist in the both of two results. The threshold of proposed vessel filter is called vascular threshold in this paper. It can be deduced that the result of extracting small nodules is determined by the two parameters: nodular threshold and vascular threshold. In experiment, the nodular threshold was tuning from 0.35 to 0.55 , and the vascular threshold was adjusted from 0.6 to 0.8 . The intervals of two thresholds are both 0.05 . Figure 4 (f) illustrates the result of subtracting false positives from Fig. 4 (c) when the vascular threshold was fixed to 0.7 . The removed false positives are given in Fig. 4 (g).

\subsection{Classification}

According to the Japanese Pneumoconiosis Law, the diagnosis of type 3-b is made by the size of nodular objects. And for the cases of type 1,2 and 3-a, the classification is performed based on the density level of small nodular objects. Therefore, we recognized the HRCT scans in two steps: 1.Classifying the cases of type 3-b and type non-3$\mathrm{b}$ (type 1,2 and 3-a) from all images; 2.Classifying the cases of type 1,2 and 3 -a.

Recognizing the cases of type 3-b is performed as follows. 1.Computation of nodule's diameter in three axis; 2.Selection of a threshold which has best performance on classifying cases of type 3-b and non-3-b in the training set; 3. Classification of cases in the test set by the threshold obtained in the step 2.

In the process of classifying cases of type 1,2 and 3-a, 


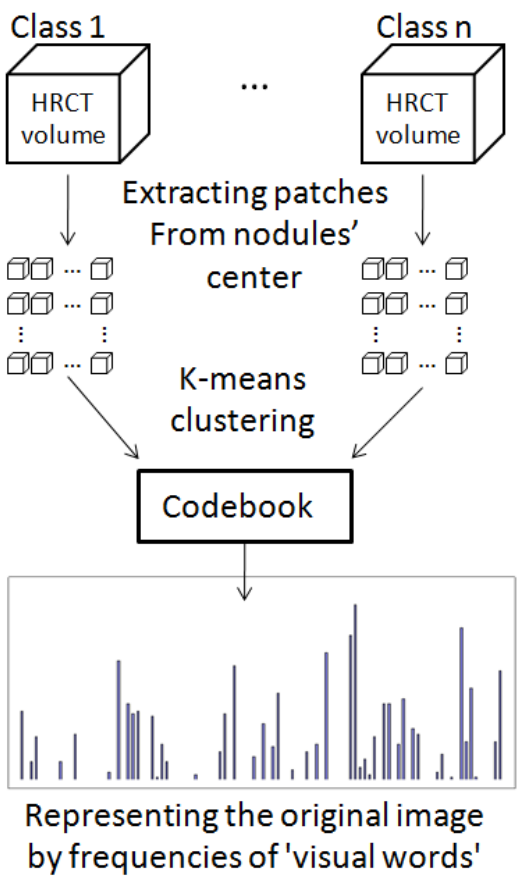

Fig. 5 Framework of bag-of-features.

a bag-of-features-based method was adopted to generate input feature vectors for the classifier. The bag-of-features is a image representation model used for object recognition in computer vision [13]. It is reported that the bag-of-features is helpful to classify various pulmonary patterns [14]-[16]. Figure 5 illustrates the framework of bag-of-features-based method: 1.Calculating local features on small patches locating on nodules' center; 2.Concentrating all feature vectors into limited number of clusters for learning a code-book; 3.Applying frequencies of 'visual words' to represent the original images and generate a histogram as input vectors for classifier.

In this study, the local features consists of three parts. One is the number of voxels belonging to nodules within the patches. Considering that it may be several parts of nodules rather than entire nodules presence in the patches, the number of voxels is better to represent the density of nodules in the local regions. The other two parts are features based on gray level co-occurrence matrix (GLCM) [17] and grey-level run lengths matrix (GLRLM) [18]. From Fig. 1, it is clearly that the intensity of lung tissues is affected by the nodules. The more nodules exist, the greater extent the tissues changes. Therefore, the statistical texture features are adopted to discriminate various nodular patterns. Table 2 and Table 3 summarize the texture features based on GLCM and GLRLM respectively. The size of patches is the parameter for this local features extraction. We considers that the relatively large-sized patches are suitable to represent the information of nodular intensity. In practice, we tried the size from $15 \times 15 \times 15$ to $40 \times 40 \times 40$ with a interval of 5 voxels.

After extracting local features from HRCT scans, the features are concentrated to a group of clusters in a high di-
Table 2 The features based on gray level co-occurrence matrix (GLCM).

\begin{tabular}{|c|}
\hline Entropy \\
\hline Energy \\
\hline Contrast \\
\hline Homogeneity \\
\hline Mean \\
\hline Variance \\
\hline Correlation \\
\hline Maximum Probability \\
\hline Inverse Difference \\
\hline Cluster Tendency \\
\hline
\end{tabular}

Table 3 The features based on run-length matrix (GLRLM).

\begin{tabular}{|c|}
\hline Short Run Emphasis \\
\hline Long Run Emphasis \\
\hline Low Gray-level Run Emphasis \\
\hline High Gray-level Run Emphasis \\
\hline Short Run Low Gray-level Run Emphasis \\
\hline Short Run High Gray-level Run Emphasis \\
\hline Long Run Low Gray-level Run Emphasis \\
\hline Long Run High Gray-level Run Maximum Emphasis \\
\hline Gray-Level Non-uniformity \\
\hline Run Length Non-uniformity \\
\hline
\end{tabular}

mensional feature space. We adopted K-means algorithm to calculate the clusters' centers in experiment. A code-book is obtained by these centers, and each center relates to a "visual word" in the code-book. When the code-book is obtained, the local features are labeled by the nearest visual word. For each case, a histogram is generated by this assignment which is used to indicate the frequency of visual words. Because the same type of cases gives similar histograms, these histograms can be adopted as input feature vector for the classifier. The number of clusters in the Kmeans is the parameter of the code-book construction. In practice the number of clusters was adjusted from 50 to 200 with a interval of 25 .

SVM was used as the classifier in experiment. We used a version named LIBSVM with RBF kernel [19]. The kernel is defined as:

$$
G\left(h, h^{\prime}\right)=\exp \left(-\gamma\left\|h-h^{\prime}\right\|^{2}\right)
$$

where $h$ and $h^{\prime}$ are both the $N$-bin histogram. The classifier includes two parameters, the soft-margin penalty $C$ and the parameter related to the RBF kernel $\gamma$. We optimized these parameters in the training stage.

The SVM classifier is essentially a binary (two-class) classifier. At present, there are two widely-used methods to extend the SVM classifier to solve the multi-class tasks: one-against-one and one-against-all [20]. The LIBSVM implement the one-against-one method for multi-class tasks. If there are $k$ numbers of categories, the classification is performed using a combination of binary SVM classifiers and a decision strategy as following: 1.Constructing $k(k-1) / 2$ SVM classifiers by samples of each pair of classes in the training stage. For example, if the pair of classes are the $i$-th class and the $j$-th class, the classifier is trained on data of the $i$-th class and $j$-th class; 2.Classifying the testing sample by the binary classifiers, and each classification is thought to be 
a voting. So $k(k-1) / 2$ votes can be obtained. For the pair of the $i$-th class and the $j$-th class, if the sample is recognized to the $i$-th class, the vote for the $i$-th class is increased by one. Otherwise, the vote for the the $j$-th class is added by one; 3 . Counting the number of votes and assigning the test sample to the class which obtain the maximum number of votes.

\section{Experiment \& Discussion}

\subsection{Data}

We obtained 175 HRCT scans of 112 different subjects from Kochi University. All scans were acquired by GE Lightspeed VCT when edge-enhanced filtering was not applied. The resolution of HRCT scans is $512 \times 512$. The slice thickness is $1 \mathrm{~mm}$, and the in-plane resolution is about $0.625 \mathrm{~mm}$. A tube voltage of $140 \mathrm{kV}$, a tube current of $250 \mathrm{~mA}$ are used. The scans were reviewed by experienced radiologists and divided into 4 types. The scans are separated into two complete independent sets by splitting each type of pneumoconiosis nearly in half. The number of scans of each type of pneumoconiosis for training and test data are summarized in Table 4. There is no crossing-subject scan between the two sets. That is to say that the scans which belongs to the same subject is put into the training or test set. The training set is adopted to train the algorithms, and the test set is used to evaluate performance of our system.

\subsection{Results}

The proposed method includes six parameters: nodular threshold, vascular threshold, size of patch, number of clusters and two parameters related to SVM classifier. In the recognition of cases of type 3-b, the nodular threshold and vascular threshold are used and fixed to 0.01 and 0.99 respectively. In the classification of type 1,2 and 3-a, all parameters are simultaneously adjusted. We used leave-oneout test to optimize parameters in the training stage. The one combination of possible values of all parameters which produce the best classification accuracy is chosen as the optimized parameter, and used to evaluate the performance of method on the test data set. For the nodular threshold and vascular threshold, we tried the values from 0.35 to 0.55 and 0.6 to 0.8 respectively. The intervals of two thresholds were both 0.05 . The range of path size was from $20 \times 20 \times 20$ to $40 \times 40 \times 40$ with a step of $5 \times 5 \times 5$. On the choice of number of clusters, we tried the number from 50 to 200 with a in-

Table 4 Number of scans for the four types of pneumoconiosis cases in the training and test set. There is no crossing-subject scans in the two sets.

\begin{tabular}{|c|c|c|}
\hline & Training data & Test data \\
\hline Type 1 & 23 & 23 \\
\hline Type 2 & 12 & 12 \\
\hline Type 3-a & 17 & 14 \\
\hline Type 3-b & 38 & 36 \\
\hline Total & 90 & 85 \\
\hline
\end{tabular}

terval of 25. We compared our method with two kinds of baseline method, Sato's [11] and Li's filter [12]. The paraments of the baseline methods and the tuning method of these paraments are same with our method.

The comparison of overall accuracy between our methods and two kinds of baseline method are given in Table 5 . It can be seen that the proposed method has the best performance. The results of classification are $90.6 \%$ (77/85) by our filter, $80.0 \%$ (68/85) by Sato's and 76.5\% (65/85) by Li's filter respectively. The confusion tables are summarized in Table 6 (our method), Table 7 (Sato's filter) and Table 8 (Li's filter) respectively. We also compared the statistical differences between the proposed method and two kinds of baseline method by McNemar's test. Since the McNemar's test is a widely-used method for assessing the statistical differences between the results of recognition method with matched pairs of subjects [21], we adopted it to analyze the responses by our filter and baseline method. The $p$ values are given in Table 9. All of the $p$ values are less than 0.05 .

Table 5 Comparison of three methods.

\begin{tabular}{|c|c|}
\hline Method & Accuracy \\
\hline Proposed & $90.6 \%$ \\
\hline Sato's & $80.0 \%$ \\
\hline Li's & $76.5 \%$ \\
\hline
\end{tabular}

Table 6 Confusion table of proposed method, overall accuracy $=90.6 \%$.

\begin{tabular}{|c|c|c|c|c|c|}
\hline & \multicolumn{4}{|c|}{ Estimated Types } & \\
\hline True Types & Type 1 & Type 2 & Type 3-a & Type 3-b & Sensitivity \\
\hline Type 1 & 23 & 0 & 0 & 0 & $100 \%$ \\
\hline Type 2 & 0 & 11 & 1 & 0 & $91.7 \%$ \\
\hline Type 3-a & 0 & 1 & 13 & 0 & $92.9 \%$ \\
\hline Type 3-b & 0 & 3 & 3 & 30 & $83.3 \%$ \\
\hline Precision & $100.0 \%$ & $73.3 \%$ & $76.5 \%$ & $100.0 \%$ & \\
\hline
\end{tabular}

Table 7 Confusion table of Sato's filter, overall accuracy $=80.0 \%$.

\begin{tabular}{|c|c|c|c|c|c|}
\hline & \multicolumn{4}{|c|}{ Estimated Types } & \\
\hline True Types & Type 1 & Type 2 & Type 3-a & Type 3-b & Sensitivity \\
\hline Type 1 & 23 & 0 & 0 & 0 & $100.0 \%$ \\
\hline Type 2 & 1 & 3 & 8 & 0 & $25.0 \%$ \\
\hline Type 3-a & 0 & 2 & 12 & 0 & $85.7 \%$ \\
\hline Type 3-b & 0 & 1 & 5 & 30 & $83.3 \%$ \\
\hline Precision & $95.8 \%$ & $50.0 \%$ & $48.0 \%$ & $100.0 \%$ & \\
\hline
\end{tabular}

Table 8 Confusion table of Li's filter, overall accuracy $=76.5 \%$.

\begin{tabular}{|c|c|c|c|c|c|}
\hline & \multicolumn{4}{|c|}{ Estimated Types } & \\
\hline True Types & Type 1 & Type 2 & Type 3-a & Type 3-b & Sensitivity \\
\hline Type 1 & 19 & 0 & 4 & 0 & $82.6 \%$ \\
\hline Type 2 & 0 & 5 & 7 & 0 & $41.7 \%$ \\
\hline Type 3-a & 2 & 1 & 11 & 0 & $78.6 \%$ \\
\hline Type 3-b & 0 & 0 & 6 & 30 & $83.3 \%$ \\
\hline Precision & $90.5 \%$ & $83.3 \%$ & $39.3 \%$ & $100.0 \%$ & \\
\hline
\end{tabular}

Table 9 Statistical differences by MCNEMAR's test.

\begin{tabular}{|c|c|}
\hline Methods & P value \\
\hline Proposed vs Sato's & 0.046 \\
\hline Proposed vs Li's & 0.016 \\
\hline
\end{tabular}




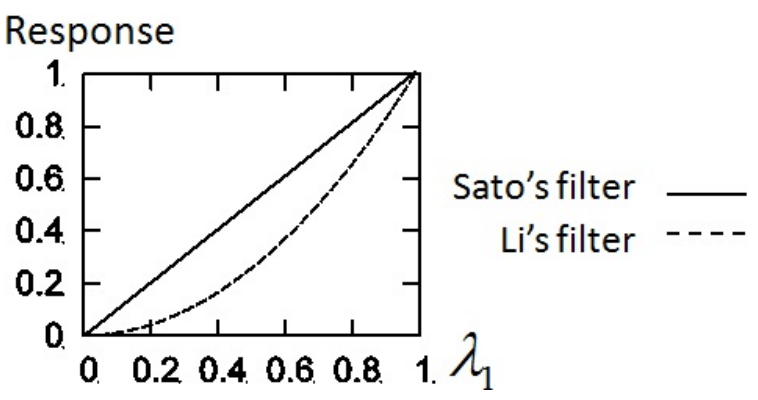

(a)

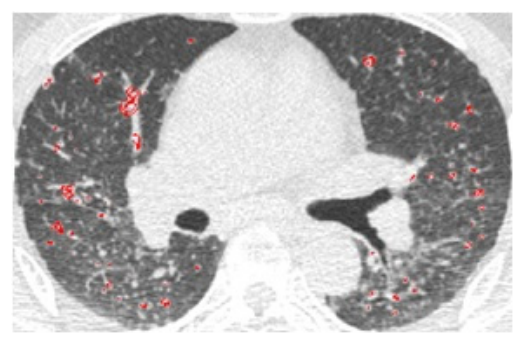

(c)

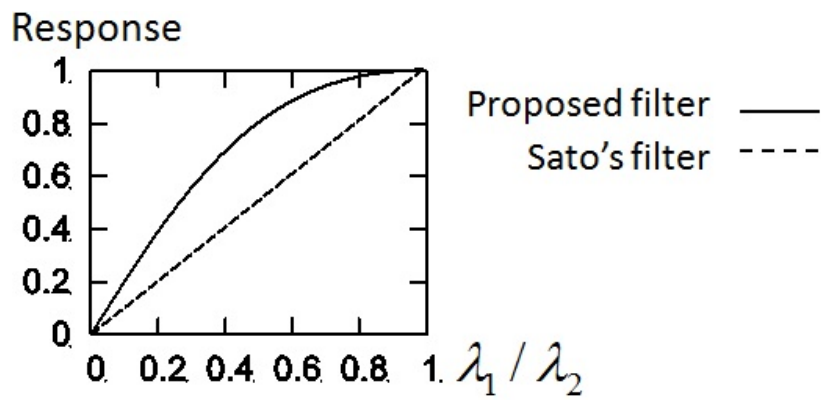

(b)

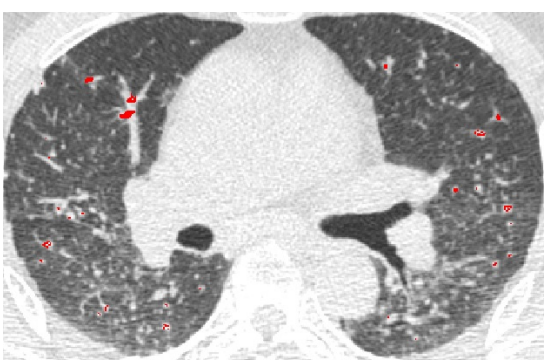

(d)

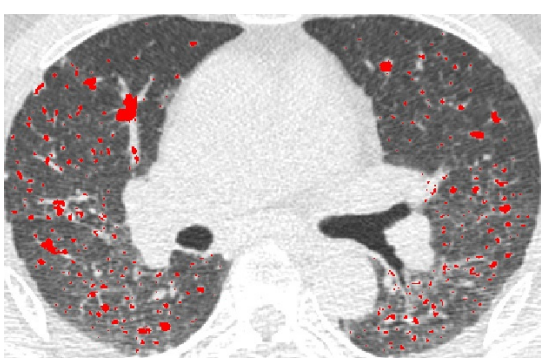

(e)

Fig. 6 Comparison of three filters: (a) Comparison of response between Sato's and Li's filter; (b) Comparison of response between proposed and Sato's filter; (c) The result of nodule extraction by Sato's filter (the nodular threshold $=0.5$ ); (d) The result of nodule extraction by Li's filter (the nodular threshold $=0.5)$; (e) The result of nodule extraction by proposed filter (the nodular threshold $=0.5$ ).

\subsection{Discussion}

According to the [12], the quality of nodule filter can be evaluated from the two aspects: sensitivity and specificity. The sensitivity means that the nodule filter should give a strong response to blob-like objects, and the specificity indicates that the nodule filter should not produce strong response to other objects, such as line-like objects. Because the conventional nodule filters have been developed to detect SPNs, they pay more attention to the question of improving discriminatory power (specificity) to distinguish nodules from other objects. But in the CAD schemes of pneumoconiosis which is featured by large numbers of nodular opacities, it is required to detect more nodules (sensitivity). The performance of these methods in detecting pneumoconiotic nodules is limited which affect the classification of pneumoconiosis. A novel filter with good sensitivity is required to detect pneumoconiotic nodules in the CAD system.

To extract more pneumoconiotic nodules, we proposed a novel approach in the study. Because all eigenvalues are approximately equal in the nodules' center, we consider that the product of differences between $\left(\lambda_{1}, \lambda_{2}\right)$ and $\left(\lambda_{2}, \lambda_{3}\right)$ is better to indicate the shape of objects and position of voxel. Although the product is also adopted in the Sato's filter, it will be simplified and not used in the computation. To avoid this simplification, we adopted an monotonically increasing function to represent the difference between eigenvalues. Figure 6(a) and Fig. 6(b) compare the response of three filters. It can be found the decreasing of response of proposed filter is slower than the two baseline methods. Therefore more nodules can be extracted, as be shown in Fig. 6(c), Fig. 6(d), Fig.6(e). The experiment results demonstrate that the increasing extracted nodules is useful for the classification of pneumoconiosis.

\section{Conclusion}

In this research, we proposed a CAD method to classify pneumoconiosis on HRCT images. Since the diagnosis of pneumoconiosis is based on the frequency of nodules, it is difficult to classify pneumoconiosis if only few nodules are extracted. We aimed to firstly extract nodules as many as possible, and then eliminate false positives. Because largesized nodules are only present in cases of type 3-b, the classification of pneumoconiosis is performed in the following steps: 1 .We extracted large-sized nodules; 2 .The cases of type 3-b and non-3-b (type 1,2 and 3-a) were classified according to the size of nodules; 3.The small-sized nodules were extracted from the cases of type non-3-b; 4 . We adopted a bag-of-features-based method to compute feature vectors on the patches locating the nodules'center and generate input vectors for an SVM classifier; 5.The cases of type 1,2 and 3-a were classified. The accuracy of classification by our method was $90.6 \%$. Comparing with the baseline filters, our method has better performance on classifying pneumoconiosis. Experiment results showed that the proposed method would be helpful for classification of pneumoconiosis. In future research, we will try to classify pneumoconiosis according to the international classification of HRCT for occupational and environmental respiratory diseases (ICOERD) [22]. 


\section{Acknowledgements}

This work was supported by MEXT KAKENHI Grant Number 21103008 .

\section{References}

[1] Ministry of health of the peopole's public of China, "Chinese Occputial Diseases in 2010," Ministry of health of the peopole's public of China, http://www.moh.gov.cn/publicfiles/business/htmlfiles/ mohwsjdj/s5854/201105/51676.htm, accessed Mar.26.2012. (In Chinese)

[2] A. Savranla, R. Altin, K. Mahmutyazicioglu, H. Ozdemir, L. Kart, T. Ozer, and S. Gundogdu, "Comparison of chest radiography and high-resolution computed tomography findings in early and lowgrade coal worker's pneumoconiosi," European Radiology, vol.5, no.2, pp.175-180, Aug. 2004.

[3] R.P. Kruger, W.B. Thompson, and A.F. Turner, "Computer diagnosis of pneumoconiosis," IEEE Trans. Syst. Man Cybern., vol.SMC-4, no.1, pp.40-49, Jan. 1974.

[4] A.F. Turner, R.P. Kruger, and W.B. Thompson, "Automated computer screening of chest radiographs for pneumoconiosis," Investigative Radiology, vol.11, no.4, pp.258-266, July-Aug. 1976.

[5] P. Yu, H. Xu, Y. Zhu, C. Yang, X. Sun, and J. Zhao, "An automatic computer-aided detection scheme for pneumoconiosis on digital chest radiographs," J. Digital Imaging, vol.24, no.3, pp.1-12, June 2010.

[6] V. Murray, M.S. Pattichis, H. Davis, E.S. Barriga, and P. Soliz, "Multiscale AM-FM analysis of pneumoconiosis X-ray images," 16th IEEE International Confference on Image Processing, Cairo, The Egypt, pp.4201-4204, Nov. 2009.

[7] E. Okumura, I. Kawashita, and T. Ishida, "Computerized analysis of pneumoconiosis in digital chest radiograph: Effect of artificial neural network trained with power spectra," J. Digital Imaing, vol.24, no.6, Dec. 2011.

[8] M.S. Ginsberg, O. Akin, D.M. Berger, M.F. Zakowski, and D.M. Panicek, "Pulmonary Tumorlets: CT Findings," American Journal of Roentgenology, vol.183, no.2, pp.293-296, Aug. 2004.

[9] K.T. Bae, J.S. Kim, Y.H. Na, K.G. Kim, and J.H. Kim, "Pulmonary nodules: Automated detection on CT images with morphologic matching algorithm-preliminary results," Radiology, vol.236, no.1, pp.286-294, July 2005.

[10] P.F. Felzenszwalb, R.B. Girshick, D. McAllester, and D. Ramanan, "Object detection with discriminatively trained part based models," IEEE Trans. Pattern Anal. Mach. Intell., vol.32, no.9, pp.16271645, Sept. 2010.

[11] Y. Sato, C. Westin, A. Bhalerao, S. Nakajima, N. Shiraga, S. Tamur, and R. Kikinis, "Tissue classification based on 3D local intensity structures for volume rendering," IEEE Trans. Vis. Comput. Graphics, vol.6, no.2, pp.160-180, April-June 2000.

[12] Q. Li, S. Sone, and K. Doi, "Selective enhancement filters for nodules, vessels, and airway walls in two- and three-dimensional CT scans," Medical Physics, vol.30, no.7, pp.1810-1820, Aug. 2009.

[13] G. Csurka, C.R. Dance, L. Fan, J. Willamowski, and C. Bray, "Visual categorization with bags of keypointm," ECCV International Workshop on Statistical Learning in Computer Vision, Prague, The Czech Republic, pp.1-22, May 2004.

[14] M.J. Gangeh, L. Sorensen, S.B. Shaker, M.S. Kamel, M.De. Bruijne, and M. Loog, "A texton-based approach for the classification of lung parenchyma in CT images," Medical Image Computing and Computer-Assisted Intervention - MICCAI 2010, vol.6363, pp.595602, Springer-Berlin, Heidelberg, 2010.

[15] R. Xu, Y. Hirano, R. Tachibana, and S. Kido, "Classification of diffuse lung disease patterns on high-resolution computed tomography by a bag of words approach," Medical Image Computing and
Computer-Assisted Intervention - MICCAI 2011, ed.G.Fichtinger, vol.6893, pp.183-190, 2011.

[16] N. Kato, M. Fukui, and T. Isozaki, "Bag-of-features approach for improvement of lung tissue classification in diffuse lung disease," Proc. SPIE, Lake Buena Vista, The USA, vol.7260, 72600C, Aug. 2009.

[17] A.S. Kurani, D. Xu, J. Furst, and D.S. Raicu, "Co-occurrence matrices for volumetric data," 7th IASTED International Conference on Computer Graphics and Imaging, Kauai, USA, pp.447-452, Aug. 2004.

[18] D. Xu, A.S. Kurani, J.D. Furst, and D.S. Raicu, "Run-length encoding for volumetric texture," 4th IASTED International Conference on Visualization, Imaging, and Image Processing, pp.534-539, Marbella, Spain, Sept. 2004.

[19] C.C. Chang and C.J. Lin, "LIBSVM: A library for support vector machines," ACM Transactions on Intelligent Systems and Technology, vol.2, no.3, pp.1-27, April 2011.

[20] C.R. Bishop, Pattern Recognition and Machine Learning, Springer, Berlin, 2006

[21] N. Japkowicz and M. Shah, Evaluating Learning Algorithms: A Classification Perspective, Cambridge University Express, Cambridge, 2011.

[22] Y. Kusaka, K.G. Hering, and J.E. Parker, International Classification of HRCT for Occupational and Environmental Respiratory Diseases, Springer, Berlin, 2005

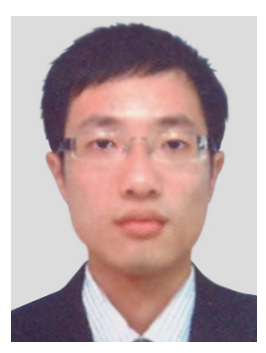

Wei Zhao received the M.S. degree in Electronic and Information System Engineering from Yamaguchi University, in 2011. He is currently a candidate for a $\mathrm{PhD}$ degree in Applied Medical Engineering Science at Yamaguchi University. His research interests include medical image processing and machine learning.

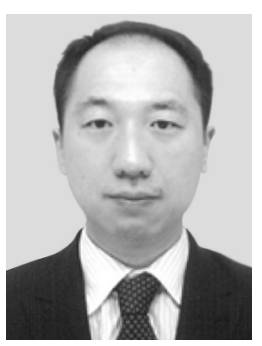

Rui Xu received the B.S. and M.S. degrees in 2001 and 2004, respectively, from School of Electronic and Information, South China University of Technology. He received the Ph.D. degree in 2007 from the Graduate School of Science and Engineering, Ritsumeikan University, Japan. He worked in Digital Technology Research Center, Sanyo Electric Co., Ltd., Japan, from 2008 to 2010. Since April 2010, he has been a research staff in Graduate School of Medicine, Yamaguchi University, Japan. His research fields are medical image processing and pattern recognition. He is a member of JAMIT (Japanese Society of Medical Imaging Technology) and IEEE. 


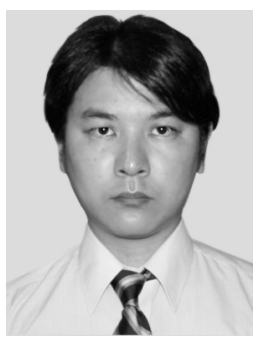

Yasushi Hirano received the B.S. and M.S. degrees in electronic informatics, and Ph.D degree in information engineering from Nagoya University in 1995, 1997 and 1999, respectively. His major fields of study are pattern recognition, image processing, parallel processing and interuniversity authentication infrastructure. He was a Research Fellow of the Japan Society for the Promotion of Science (PD) in 1999-2000, an assistant professor at Department of information engineering, Nagoya University in 2000-2002, assistant professor at Information Technology Center, Nagoya University in 2002-2004, and an associate professor at Information Technology Center, Nagoya University in 2004-2010. He has been an associate professor at Graduate School of Medicine, Yamaguchi University since 2010. He is a member of IEEE, JAMIT (Japanese Society of Medical Imaging Technology), and JSMBE (Japanese Society for Medical and Biological Engineering).

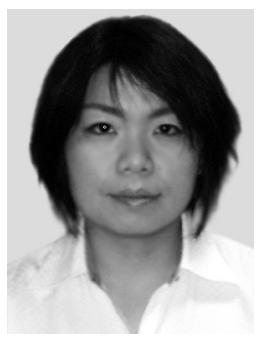

Rie Tachibana received her M.S. and Ph.D. degrees in System Engineering from Yamaguchi University, Japan, in 2003 and 2006, respectively. She is currently a lecturer in the Department of Information Science and Technology, Oshima National College of Maritime Technology, Japan. Her research interests include medical image processing and computer-aided diagnosis.

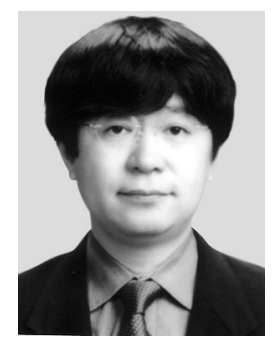

Shoji Kido received his M.D degree from Osaka University in 1988. He received his Ph.D. degrees in Medicine and Information Science from Osaka University in 1992 and 1999, respectively. He was a staff radiologist in Yao Municipal Hospital, Nishinomiya Municipal Hospital, and Osaka Medical Center for Cancer and Cardiovascular Diseases from 1992 to 1999 . He was a Professor in the Department of Computer Science and Systems Engineering at Yamaguchi University from 1999 to 2004 . He is currently a Professor in the Department of Applied Medical Engineering Science, Graduate School of Medicine, Yamaguchi University.

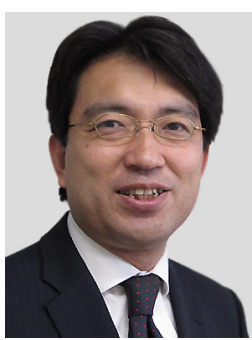

Narufumi Suganuma trained as an internist and then in epidemiology at the Okayama University and University of London (distant learning). His research interests include the radiological classification of occupational lung diseases, lung cancer CT screening, tumor marker of occupational cancer, prevention of allergic diseases with technological intervention. $\mathrm{He}$ is one of the ILO experts on the ILO International Classification of Radiograph of Pneumoconioses and teaches its application in Asian countries. He is currently Professor and Chief of Environmental Medicine, Kochi Medical School. He holds an US NIOSH B reader Certificate. 